\begin{tabular}{lll}
\hline AECORDS OF PHARMACEUTICAL \\
AND BIOMEDICAL SCIENCES
\end{tabular}

\title{
Lamivudine: An antiviral drug with high risk factor for selection of resistance in $\mathrm{HBV}$ patients
}

\author{
Mahmoud A. Abdel Aal ${ }^{1 *}$, Dina M. Khodeer ${ }^{1}$, Nader A. Nemr ${ }^{2}$, Mona F. El-Azab ${ }^{1}$ \\ ${ }^{1}$ Department of Pharmacology \& Toxicology, Faculty of Pharmacy, Suez Canal University, Ismailia 41522, \\ Egypt, ${ }^{2}$ Infectious Diseases Department, Faculty of Medicine, Suez Canal University, Ismailia, Egypt
}

Received on: 30. 06.2021

Revised on: 30. 08.2021

Accepted on: 03. 09. 2021

*Correspondence Author:

Tel: +2-012-73219991

E-mail address:

mahmoudahmed11099@yahoo.com

\begin{abstract}
Globally, it has been reported that nearly 257 million people suffer from chronic Hepatitis B virus (HBV). In Egypt, HBV has been classified as a moderate endemic where $4 \%$ of the population presented with chronic HBV. Long term Lamivudine (LMV) monotherapy has been found to develop resistant strains of $\mathrm{HBV}$ where the polymerase gene specifically is mutated (i.e., YMDD as a primary mutation). This review aimed to determine the correlation between LMV therapy and development of resistance in chronic HBV patients and to investigate the genes responsible for LMV resistance. LMV as an antiviral therapy has a fair response rate in the studied patients. Unfortunately, the long duration treatment with LMV leads to development of LMV resistant mutations altering the efficacy of the drug.
\end{abstract}

Keywords: HBV, Lamivudine, YMDD, Mutation, Resistance.

\section{Introduction}

Hepatitis B virus (HBV) is a $3.2 \mathrm{~kb}$, double stranded, relaxed circular DNA (rcDNA) virus. Its forward strand DNA is not complete and is shorter than its reverse strand (Wei and Ploss, 2020). As a result, after entering hepatitis cells, HBV DNA begins a conversion to covalently closed circular DNA (cccDNA), and this cccDNA is the template for messenger RNA used in translating viral protein. In addition, the cccDNA is also the template for pre-genomic RNA in the production of additional rcDNA through reverse transcription by $\mathrm{HBV}$ polymerase. The HBV polymerase lacks a conventional proofreading function, and it has a highly powerful replication function that can produce 1011 viruses/day ((Tsukuda and Watashi, 2020). As a result, HBV has a high spontaneous mutation rate, and in an environment of incomplete viral suppression via antiviral therapy, the selection of resistance promoting mutations is facilitated.

Over 257 million people are chronically infected with $\mathrm{HBV}$ worldwide. Furthermore, $\mathrm{HBV}$ is considered moderately endemic in Egypt with 4\% of the population having evidence of chronic HBV infection (Hutin et al., 2018). Despite advances in vaccination and treatment, $\mathrm{HBV}$-related deaths due to liver cirrhosis or hepatocellular carcinoma (HCC) increased between 1990 and 2013 globally by 33\% (686,000 cases in 2013) (Ginzberg et al., 2018). Therefore, the main treatment goal for chronic hepatitis B is to suppress viral replication and consequently to prevent progression of the disease (EASL, 2017).

In most cases, this is achieved by the orally available nucleotide analogues that act as 
competitive inhibitors of the HBV reverse transcriptase, as their incorporation into the DNA strand provokes chain termination and hence suppresses HBV replication. One of the major problems with using nucleotide analogues has been the development of drug resistance mutations during longterm therapy (Tacke and Kroy, 2016).

Additionally, CHB infections are treated by using six approved antiviral agents. The antiviral agents include interferon (conventional and pegylated), and five nucleotide analogues (NAs), viz. lamivudine (LMV), adefovir (ADV), telbivudine (LdT), entecavir (ETV) and tenofovir (TDF) (Chantratita et al., 2016). The Antiviral therapy with LMV, ADV or LdT was shown to be a major risk factor for selection of resistance. Worldwide, eighty percent of patients showed a development of LMV resistant strains after five years of treatment with LMV alone (Kim et al., 2014).

Moreover, Out of $10 \mathrm{RT}$ codons $(80,173,180,181$, 184,194, 202, 204, 236 and 250), the rtA181V, $\mathrm{rtM} 204 \mathrm{~V} / \mathrm{I}$, rtN236T and $\mathrm{rtM} 250 \mathrm{~V}$ are the more prevalent mutations found in the drug resistant HBV strains (Chantratita et al., 2016). Notably, mutations in the HBV polymerase open reading frame (P-ORF) related to drug resistance may alter the amino acid sequence of the surface antigen proteins $(S)$, because of the overlapping reading frames of the $\mathrm{P}$ and $\mathrm{S}$ gene (Shirvani-Dastgerdi et al., 2017).

\section{Chronic Hepatitis B}

\subsection{Epidemiology of HBV}

Around $30 \%$ of the world's population shows serological evidence of current or past HBV infection (Hutin et al., 2018). About half the total liver cancer mortality in 2010 was attributed to HBV infection, and from 1990 to 2010, the worldwide mortality associated with liver cancer increased by $62 \%$ and that associated with cirrhosis increased by 29\% (Trépo et al., 2014).

\subsection{Virology}

HBV belongs to the Hepadnaviridae family. It is a partly double stranded DNA virus. The transcriptional template of $\mathrm{HBV}$ is the cccDNA, which resides inside the hepatocyte nucleus as a mini chromosome. The maintenance of cccDNA is essential for the persistence of the virus.
The replication of HBV implicates reverse transcription of the pregenomic RNA intermediate into HBV DNA. Reverse transcriptase is error prone and the mutation rate is high. The receptor for HBV entry into hepatocytes is sodium taurocholate polypeptide (Tsukuda and Watashi, 2020).

\subsection{Management}

The main goal of therapy for patients with chronic HBV infection is to improve survival and quality of life by preventing disease progression, and consequently HCC development. Additional goals of antiviral therapy are to prevent mother to child transmission, hepatitis $\mathrm{B}$ reactivation and the prevention and treatment of $\mathrm{HBV}$-associated extrahepatic manifestations (EASL, 2017).

\section{Hepatitis B Virus Mutations}

\subsection{HBV S-Gene Mutants}

The pre-S1/S2/S ORFs encode three envelope proteins (large, middle and small) which are determinant for virus assembly and virus attachment to hepatocytes. L protein (pre-S1 domain) is the substrate for viral receptor attachment; $\mathrm{M}$ protein (pre-S2 domain) function is not well understood and, finally, $S$ protein ( $S$ domain) is commonly referred to as the HBsAg or Australian antigen. The small, the middle and the large proteins are detected as $\mathrm{HBsAg}$. $\mathrm{HBsAg}$ protein contains the major B cell epitope, the "a" determinant (121-149 aa) (Caligiuri et al., 2016).

\section{Lamivudine}

\subsection{Drug Profile}

Lamivudine ((-) 28-deoxy-38-thiacytidine, 3TC) is a member of a class of antiviral nucleoside analogs; Lamivudine is an analogue of cytidine, that inhibit hepadnavirus replication specifically by terminating viral DNA synthesis (Allen et al., 1998). LMV can be taken orally as a liquid or tablet and can take with or without food (The American Society of Health, 2016). It is safe for people over three months of age and can be used during pregnancy. LMV was first approved for use in the United States in 1995 (Awofisayo et al., 2019). 
LMV is administered by mouth, and it is rapidly absorbed with a bio-availability of over $80 \%$. Some research suggests that lamivudine can cross the blood-brain barrier. LMV showed no evidence of carcinogenicity or mutagenicity in in vivo studies in mice and rats at doses from 10 to 58 times those used in humans also; its half-life is 5-7 hours in adults (Reis et al., 2013).

\subsection{Mechanism of Action}

As mentioned before, Lamivudine is an analogue of cytidine. It can inhibit the reverse transcriptase of hepatitis B virus. It is phosphorylated to active metabolites that compete for incorporation into viral DNA. They act as a chain terminator of DNA synthesis. The lack of a 3'-OH group in the incorporated nucleoside analogue prevents the formation of the 5' to $3^{\prime}$ phosphodiester linkage essential for DNA chain elongation, and therefore, the viral DNA growth is terminated (Torresi and Locarnini, 2000).

\subsection{Lamivudine Resistance}

Long-term LMV monotherapy selects for resistant HBV strains harboring specific mutations in the polymerase gene (i.e., M204I/V as a primary mutation and L80, L180M, V173L as the most relevant secondary mutations) at rates that increase from $20 \%$ after 1 year to a peak of $70 \%$ after 5 years of drug therapy. LMV resistance was first described in the YMDD (tyrosine-methionineaspartate-aspartate) locus of the HBV reverse transcriptase gene. Because the risk of developing LMV resistance (LMV-R) directly correlates with a slow response of serum HBV DNA at month 6, incomplete suppression of HBV replication seems to play a key role in the generation of mutated strains, as suggested with other antiviral regimens (Lampertico et al., 2011).

\subsection{Treatment of Resistance}

The clinical management of resistance (NUC-R) varies according to the rescue drug (nucleoside vs. nucleotide), the rescue strategy (switch vs. add-on), and timing of rescue (early vs. late). The drug of choice is selected according to the in vitro crossresistance profile of the regimen. Nucleoside analogues such as LMV, LdT, and ETV share a similar resistance profile characterized by mutation in position 204, accompanied by several compensatory changes in nucleotides. Conversely, the resistance pattern of the nucleotide analogue ADV differs significantly, because it is based on changes at position 236 and 181 (i.e., N23T and A181V). TDF has a predicted resistance pattern similar to ADV, although not yet identified (Fung et al., 2017).

As a general rule, a nucleotide analogue is recommended as rescue for $\mathrm{HBV}$ resistance in patients exposed to nucleoside analogues, whereas a nucleoside analogue is recommended for nucleotide-related resistance. TDF (or ADV if TDF is not available) is the nucleotide of choice for the rescue of nucleoside-resistant patients (Fung et al., 2017). Conversely, in the presence of ADV resistance (ADV-R), switching to TDF and adding a second drug is recommended; after occurrence of TDF resistance (TDF-R), the addition of a nucleoside is recommended (EASL, 2017).

\section{Conclusion}

It could be concluded that chronic hepatitis B, has a relatively benign course in Egypt, however regular follow up is recommended due to its dynamic nature and for early detection of its devastating complications. Lamivudine as a 1st line antiviral therapy has a fair response rate in the studied region that can be used as 1st line regimen for those with active hepatitis and even those with cirrhosis, unfortunately the long duration treatment with Lamivudine develops Lamivudine resistant mutations alter the efficacy of the drug. Sequencing of HBV samples showed YMDD mutation substitution of methionine at amino acid position204 to isoleucine (rtM204I, YIDD mutant).

\section{References:}

Allen, M. I., M. Deslauriers, C. W. Andrews, G. A. Tipples, K.-A. Walters, D. L. Tyrrell, N. Brown, G. Lamivudine Clinical Investigation and L. D. Condreay (1998). "Identification and characterization of mutations in hepatitis B virus resistant to lamivudine." Hepatology 27(6): 16701677.

Awofisayo, S. O., M. I. Arhewoh and A. O. Okhamafe (2019). "Effect of Food/Beverage and Selected Drugs on the Oral Absorption of Artemether-Lumefantrine Tablet: an in situ and in vivo Study." Bulletin of Faculty of Pharmacy, Cairo University 57(2): 137-147. 
Caligiuri, P., R. Cerruti, G. Icardi and B. Bruzzone (2016). "Overview of hepatitis B virus mutations and their implications in the management of infection." World journal of gastroenterology 22(1): 145-154.

Chantratita, W., K.-S. Song, V. Pongthanapisith, N. Thongbaiphet, K. Angkanavin, S. B. Nimse, M. D. Sonawane and T. Kim (2016). "HBV/4DR 9G test and its comparison with INNO-LiPA HBV multiDR test for the detection of drug-resistant Hepatitis B virus." Journal of Virological Methods 237: 5863.

EASL (2017). "EASL 2017 Clinical Practice Guidelines on the management of hepatitis B virus infection." Journal of Hepatology 67(2): 370-398.

Fung, S., P. Kwan, M. Fabri, A. Horban, M. Pelemis, H.-W. Hann, S. Gurel, F. A. Caruntu, J. F. Flaherty and B. Massetto (2017). "Tenofovir disoproxil fumarate (TDF) vs. emtricitabine (FTC)/TDF in lamivudine resistant hepatitis B: A 5year randomised study." Journal of hepatology 66(1): 11-18.

Ginzberg, D., R. J. Wong and R. Gish (2018). "Global HBV burden: guesstimates and facts." Hepatology International 12(4): 315-329.

Hutin, Y., M. Nasrullah, P. Easterbrook, B. Dongmo Nguimfack, E. Burrone, F. Averhoff and M. Bulterys (2018). "Access to treatment for hepatitis B virus infection-Worldwide, 2016." American Journal of Transplantation 18(10): 2595 2598.

Kim, J. H., Y. K. Park, E.-S. Park and K.-H. Kim (2014). "Molecular diagnosis and treatment of drugresistant hepatitis B virus." World journal of gastroenterology 20(19): 5708-5720.

Lampertico, P., M. Viganò and M. Colombo (2011). "Current management of HBV antiviral drug resistance." Current Hepatitis Reports 10(2): 120127.
Reis, J. M., A. B. Dezani, T. M. Pereira, A. Avdeef and C. H. R. Serra (2013). "Lamivudine permeability study: A comparison between PAMPA, ex vivo and in situ Single-Pass Intestinal Perfusion (SPIP) in rat jejunum." European Journal of Pharmaceutical Sciences 48(4): 781-789.

Shirvani-Dastgerdi, E., B. Y. Winer, T. CeliàTerrassa, Y. Kang, D. Tabernero, E. Yagmur, F. Rodríguez-Frías, J. Gregori, T. Luedde, C. Trautwein, A. Ploss and F. Tacke (2017). "Selection of the highly replicative and partially multidrug resistant rtS78T HBV polymerase mutation during TDF-ETV combination therapy." Journal of Hepatology 67(2): 246-254.

Tacke, F. and D. C. Kroy (2016). "Treatment for hepatitis B in patients with drug resistance." Annals of translational medicine 4(18): 334-334.

Torresi, J. and S. Locarnini (2000). "Antiviral chemotherapy for the treatment of hepatitis $b$ virus infections." Gastroenterology 118(2, Supplement): S83-S103.

Trépo, C., H. L. Y. Chan and A. Lok (2014). "Hepatitis B virus infection." The Lancet 384(9959): 2053-2063.

Tsukuda, S. and K. Watashi (2020). "Hepatitis B virus biology and life cycle." Antiviral Research 182: 104925 .

Wei, L. and A. Ploss (2020). "Core components of DNA lagging strand synthesis machinery are essential for hepatitis B virus cccDNA formation." Nature Microbiology 5(5): 715-726. 